Result. Overall, the simulation project met its pre-determined objectives of increasing confidence and competence in the medical workforce in the context of COVID-19 and physical health. Development and delivery of the workshop was rapid, with request to delivery taking 4 days.

A summary of the key lessons include:

An existing simulation faculty within the trust was essential, allowing for rapid identification of key stakeholders and those able to deliver the project.

A "direct-line" relationship to senior leadership enabled the project to be dynamic and responsive to changing demands as COVID-19 guidelines and objectives evolved.

Redeploying higher trainees with SBE experience to develop the project as a focussed team allowed for rapid delivery which was resource-effective.

The workforce found reassurance from understanding what was not expected of them, as much as what was. For example, making clear that Arterial Blood Gases would not be introduced to the psychiatric setting.

There is an ongoing learning need for physical health training through SBE in non-covid scenarios.

SBE can be an effective intervention for a range of medical grades and covering a large geographical area.

There are opportunities for developing multi-disciplinary training on physical health in psychiatry.

Conclusion. We have outlined some of the key learning outcomes from a successfully implemented SBE project during the first COVID-19 wave in spring 2020. The project has cemented the role of the relatively new simulation faculty within the trust and highlighted the effectiveness of close collaboration between leadership and a small, dedicated group of facilitators. The project has continued to be used for training new staff members and the resources have been widely shared, used by other NHS trusts and also internationally.

\section{Psychoeducation for the Frontline: Ealing Liaison Psychiatry Service (ELPS) pilot training day for London Ambulance Service (LAS)}

\author{
Emma McLean ${ }^{1 \star}$ and Mariam Alexander ${ }^{2}$ \\ ${ }^{1}$ Imperial College Healthcare NHS Trust and ${ }^{2}$ Ealing Liaison \\ Psychiatry Service, West London NHS Trust \\ ${ }^{*}$ Corresponding author.
}

\section{doi: 10.1192/bjo.2021.416}

Aims. To host the first ELPS training day specifically for LAS staff to improve their knowledge and understanding about mental health issues and the role of ELPS.

On average 13,000 calls are received by LAS relating to mental health issues every month. Many patients seen by ELPS will have multiple interactions with LAS. ELPS has previously held training for the Emergency Department team but this innovative day was designed to extend this training commitment to pre-hospital clinicians

Method. LAS training needs were initially assessed by a bespoke questionnaire and ELPS attending another LAS training event held by the new mental health joint response car team.

We then developed a training programme to match the identified training needs and which utilised the specific expertise of individual ELPS staff.

14 members of the local LAS stations attended including both Paramedics and Emergency Ambulance Clinicians. The presentations covered mental state examination, suicide, risk assessment, substance misuse, legal frameworks and then a 'challenging cases' session to bring it all together.
Pre and post course questionnaires were completed by participants, exploring attitudes and knowledge.

Result. There was a statistically significant improvement in the average self-ratings for all of the categories assessed including attitudes to mental health, confidence in assessment and knowledge relating to the process the patient will experience in the emergency department.

The knowledge about the pathway and role of liaison psychiatry showed the greatest improvement with an average 4.25 increase in pre and post course rating.

Almost all participants (9.2/10) would recommend this training day to a colleague

Conclusion. We met our objective of improving LAS staff knowledge and understanding about mental health issues and the role of ELPS. We plan to build on this successful pilot and expand our training programme for LAS with the ultimate aim of improving patient care.

The Malaysian Northern Stars (supervision, training, and reflective system) project: a multi-facet ecosystem of producing local talents

Noor Melissa Nor Hadi ${ }^{1 *}$ and Jiann Lin Loo $^{2}$

${ }^{1}$ Hospital Tuanku Fauziah and ${ }^{2}$ Ysbyty Maelor Wrecsam, Betsi

Cadwaladr University Health Board

${ }^{*}$ Corresponding author.

doi: 10.1192/bjo.2021.417

Aims. The MRCPsych (Membership of the Royal College of Psychiatrists, United Kingdom) parallel training pathway has been introduced in Malaysia to produce competent psychiatrists to deliver evidence-based psychiatric care. Certain training centres faced specific challenges during the process of implementation, including the lacking of supervisors with experience in the MRCPsych examination, over-reliance on self-study and existing continuous medical education (CME), logistic difficulty in accessing specific training courses, the sustainability of local training, and loss of manpower due to frequent mobilisation of trainees. This article is aimed to illustrate the Northern STARS (Supervision, Training, and Reflective System) project, i.e. a project implemented as a solution for those challenges and an effort to develop a sustainable model of training for the local talents in Perlis, a northern state in Malaysia.

Method. The Northern STARS initiatives included: setting up a library with more MRCPsych-related materials; introducing trainees to virtual MRCPsych support groups; organizing both physical and virtual training locally, collaborating with local and international experts for consultation and teaching, and the introduction of protected study time. Virtual platforms were used innovatively to minimise cost. Ongoing data were collected for programme evaluation and quality improvement. Trainees were actively involved in the process to facilitate the development of leadership and administrative skills.

Result. A total of seven courses covering both skill and theory training had been organised: Ultra-brief Psychological Intervention Workshop, Dialectic Behavioural Therapy workshop, Personality Disorder Workshop, Critical Appraisal Workshop, MRCPsych Lecture Series, Addiction Psychiatry Lecture, and Basic Revision Course on Electroconvulsive Therapy. An estimated amount of twenty thousand Malaysian Ringgit had been generated and channelled into the community mental health centre, accounting for the indirect cost of a subscription to ZoomTM and the intangible cost of labour effort. Overall feedback revealed a high level of satisfaction together with some specific suggestions on areas of 
improvement, including the timing of course and coverage of the curriculum. To date, six medical officers are pursuing this pathway with three of them passing one paper and another two pursuing the final part.

Conclusion. The Northern STARS project is an ecosystem of training solutions while generating income and producing more local talents to expand this project further. More long-term evaluation from the perspective of human resource and health economics can be considered to understand the efficiency of the current initiative.

\section{Peer mentoring in psychiatry: a trainee-led initiative}

Zoe Moore $^{1 \star}$, Linda Irwin ${ }^{2}$, Stuart Brown ${ }^{1}$, Julie Anderson ${ }^{2}$ and Stephen Moore ${ }^{3}$

${ }^{1}$ Belfast Health and Social CareTrust; ${ }^{2}$ Northern Health and Social Care Trust and ${ }^{3}$ South Eastern Health and Social Care Trust ${ }^{*}$ Corresponding author.

doi: 10.1192/bjo.2021.418

Aims. Our aim was to establish a Peer Mentoring Network within Psychiatry Training in Northern Ireland.

Recognising that starting a new job can be a stressful time in any junior doctor's career, we wanted to ensure that new Core Trainees (CT1s) joining our Specialty Programme were well supported through this transition.

Although Clinical and Educational Supervision is well established in providing a support structure for trainees, we believed that a peer mentoring relationship, (with allocation of a Higher Psychiatry Trainee as mentor), would be of additional benefit.

It was hoped that the scheme would prove mutually beneficial to both mentee and mentor.

Method. We delivered a presentation at CT1 induction and sent out follow-up emails to encourage participation. Higher trainees were also sent information via email and asked to complete a basic application form if interested in becoming a mentor. Prospective mentors then attended a one-day training session.

Two lead mentors, (also higher trainees), were allocated to oversee the scheme, with additional supervision from two lead Consultants. Mentor-Mentee matches were made based on information such as location, sub-specialty affiliations and outside interests.

Matched pairs were advised about the intended frequency and nature of contacts. Check-in emails were sent halfway through the year and feedback evaluations completed at the end.

Result. 95\% of trainees who completed the evaluations said they would recommend the scheme to colleagues.

Mentees reported benefits in terms of personal and professional development, whilst mentors reported improved listening, coaching, and supervisory skills.

A small number of trainees highlighted that 6 monthly rotations impacted on ability to maintain face to face contacts.

Recruitment and engagement have improved annually. We are currently running the third year of the scheme and have achieved $100 \%$ uptake amongst CT1s and are over-subscribed with mentors, (19 mentors to 13 mentees).

Conclusion. The majority of feedback received has been positive and interest in the scheme continues to grow.

Potential issues relating to location of postings has been overcome, at least in part, by recent changes to ways of working and the use of alternative forms of contact, such as video calling.

Having exceeded demand in terms of mentor recruitment, we hope to extend the scheme to include trainees of other grades, and particularly those who are new to Northern Ireland.
We are excited to see where the next stage of our journey takes us and hope that others will be inspired to embark on similar schemes within their areas of work.

Quantitative and qualitative analysis of feedback from The Psychiatry Teaching Programme for Foundation Year doctors rotating through Pennine acute trust from 2010 to 2020

Angel Namuddu ${ }^{1 *}$, Margaret Gani ${ }^{2}$ and Sarah Burlinson ${ }^{2}$

${ }^{1}$ Manchester University NHS Foundation Trust and ${ }^{2}$ Search Results Pennine Care NHS Foundation Trust

${ }^{*}$ Corresponding author.

doi: 10.1192/bjo.2021.419

Aims. To monitor the year on year trend of feedback scores regarding content, presentation and relevance of sessions delivered as part of the programme by analysing the average Likert scales. To review the confidence post topic from FY feedback. To review qualitative data on the written feedback annually using a word cloud.

Method. Collated data from teaching programme from the various teaching sessions from the past decade and analysed previous teaching reports completed by previous ST leads.

Result. Finding: Relevance: Improvement in the average score year on year, highest in 2018/19 at 4.8/5

Content: Improvement in the average score year on year, highest in 2018/19 at 4.6/5.

Delivery: Improvement in the average score year on year, highest in 2018/19 at 4.6/5.

Qualitative analysis showed that the common themes that were commented on as positives for the session were: interactive, relevant and interesting, for areas for improvements the common themes were: more interaction, split into shorter sessions, faster pace and the need practical advice

Conclusion. Recommendations: teaching for FYs should aim to be interactive, relevant and interesting and include practical advice, be shorter and faster paced. Teaching programme organisers to contine to use the foundation year feedback to improve the teaching programme including advising future trainees and organising different topics.

Demystifying the pathway of assessment and treatment for bipolar disorder - utilising co-production and algorithms to personalise the approach

Jessica Nicholls-Mindlin ${ }^{1 *}$, Angus McLellan², David Gee², Lauren Fuzi ${ }^{2}$, Matthew Taylor ${ }^{3}$ and Digby Quested ${ }^{3}$

${ }^{1}$ University of Oxford; ${ }^{2}$ Oxford Health NHS Foundation Trust and

${ }^{3}$ Oxford Health NHS Foundation Trust, Department of Psychiatry,

University of Oxford

${ }^{\star}$ Corresponding author.

doi: 10.1192/bjo.2021.420

Aims. To develop an evidence based, patient centred treatment pathway for people experiencing symptoms of bipolar disorder (BD), modifiable to include local resources.

Method. This project was developed in line with current approaches to service development such as coproduction, with patient and public involvement (PPI) and enhancing personalisation of treatment in medicine. As part of a local initiative, a multidisciplinary team was brought together to understand and analyse the current local pathway for those affected by BD. It was found 